\title{
Best serum biomarker combination for ovarian cancer classification
}

\author{
Hye-Jeong Song ${ }^{2 *}$, Eun-Suk Yang ${ }^{1,2}$, Jong-Dae Kim ${ }^{1,2}$, Chan-Young Park ${ }^{1,2}$, Min-Sun Kyung ${ }^{3}$ and Yu-Seop Kim ${ }^{1,2}$
}

From International Conference on Biomedical Engineering Innovation (ICBEI) 2016 Taichung, Taiwan. 28 October-1 November 2016

*Correspondence:

hjsong@hallym.ac.kr

2 Bio-IT Research Center,

Hallym University,

Chuncheon, South Korea

Full list of author information

is available at the end of the article

\begin{abstract}
Background: Screening test using CA-125 is the most common test for detecting ovarian cancer. However, the level of CA-125 is diverse by variable condition other than ovarian cancer. It has led to misdiagnosis of ovarian cancer.
\end{abstract}

Methods: In this paper, we explore the 16 serum biomarker for finding alternative biomarker combination to reduce misdiagnosis. For experiment, we use the serum samples that contain 101 cancer and 92 healthy samples. We perform two major tasks: Marker selection and Classification. For optimal marker selection, we use genetic algorithm, random forest, T-test and logistic regression. For classification, we compare linear discriminative analysis, K-nearest neighbor and logistic regression.

Results: The final results show that the logistic regression gives high performance for both tasks, and HE4-ELISA, PDGF-AA, Prolactin, TTR is the best biomarker combination for detecting ovarian cancer.

Conclusions: We find the combination which contains TTR and Prolactin gives high performance for cancer detection. Early detection of ovarian cancer can reduce high mortality rates. Finding a combination of multiple biomarkers for diagnostic tests with high sensitivity and specificity is very important.

Keywords: Marker selection, Classification, Ovarian cancer, Logistic regression, CA-125

\section{Background}

Ovarian cancer is the eighth most common cancer and has the fifth fatality-to-case ratio in United States. It is also undetected until it goes at late stage. According to a statistics of Centers for Disease Control and Prevention (CDC) in 2012, about 20 thousands women in United States were diagnosed with ovarian cancer, and about 75\% died from it. In addition, when ovarian cancer is found in its early stage, the probability of 5-year survival yields up to $92 \%$. However the early detection rate is only $19 \%$. It is clarify that the early detection of ovarian cancer improves the clinical output $[1,2]$.

For early diagnosis, many researches have been performed: finding multiple biomarkers [3], early detection using menopausal information [4], and finding optimal

(c) The Author(s) 2018. This article is distributed under the terms of the Creative Commons Attribution 4.0 International License (http://creativecommons.org/licenses/by/4.0/), which permits unrestricted use, distribution, and reproduction in any medium provided you give appropriate credit to the original author(s) and the source, provide a link to the Creative Commons license, and indicate if changes were made. The Creative Commons Public Domain Dedication waiver (http://creativecommons.org/publi cdomain/zero/1.0/) applies to the data made available in this article, unless otherwise stated. 
combination using machine learning algorithms $[3,5]$. Specifically, many of them have been developed for distinguishing between benign and cancer [3-7].

One of the most popular screening test for ovarian cancer is CA-125 blood test. CA-125 is a protein in the blood. The level of CA-125 is high from many women with ovarian cancer. CA-125 is also useful for guiding treatment to patients with ovarian cancer, since a high level of CA-125 decrease if treatment is working well [8-10].

However, checking CA-125 level has led to misdiagnosis of ovarian cancer. The problem with using CA-125 for screening test for ovarian cancer is that common conditions other than cancer can also cause the level of CA-125 high. In addition, someone who has ovarian cancer does not have high level of CA-125.

For detecting ovarian cancer, in previous studies, we shows the multiple biomarker has high performance rather than single biomarker [3-7].

In this paper, the goal of our experiment is to find alternative biomarker combination which shows high diagnosis performance, using a variety of machine learning techniques instead of CA-125. We perform two major tasks. Each task describes as follows:

1. We search the optimal marker combinations from 16 serum biomarker. Using 4 different algorithms, we select the best combination from each combination set.

2. We compare the methods, which is widely used for classification, of optimal combination for distinguishing normal and cancer samples.

\section{Methods}

Serum samples were obtained from 101 patients with ovarian cancer and from 92 healthy women provided through Hallym University Chuncheon Sacred Heart Hospital. To validate our approach, we do not care about cancer stage, and the state of menopause which is important factor associated with the risk of malignancy [11, 12]. The 16 serum biomarkers, which is commonly discussed for ovarian cancer researches, are used to our experiment [12-14].

To select optimal marker combination which can diagnose cancer and normal data, we use four algorithms: random forest (RF) [15], genetic algorithm (GA) [16], T-test and logistic regression (LR) [17]. The size of combination is set from 2 to 4 for reducing a time consuming. The top marker combinations for each algorithm are computed to fivefold cross-validation. We repeated it 1000 times in order to decrease the deviation of the result. The final best marker combinations are selected to average receiver operating characteristic (ROC) Area Under the Curve (AUC). ROC AUC is described on the next subsection in a detail.

With the selected optimal marker sets, for each combination size, we apply the three method, which is commonly used for classification: linear discriminant analysis (LDA) [18], K-nearest neighbor (KNN) [19] and logistic regression (LR). We compare the accuracy for classification between normal and cancer data.

\section{Receiver operating characteristic area under the curve (ROC AUC)}

In order to assess the test performance, sensitivity and specificity is commonly used and through two indicators, we can find how well a classifier can distinguish between 
patients and healthy people. When a certain diagnosis system is used, sensitivity is a measure that how well the system distinguish the samples, which is associated with condition. Specificity is a measure that how well the system distinguish the samples, which does not have associated condition. In addition, ROC curve is widely used to determine the accuracy of diagnosis $[20,21]$.

ROC is a plot that illustrates the performance of a binary classifier. In a plot, the $\mathrm{x}$-axis indicates 1-specificity and y-axis presents sensitivity. The accuracy of diagnosis is measured to AUC. Figure 1 shows the ROC graph for a settings of the decision criterion. According to AUC value, the quality of test is classified. The numbers on the curve present the degree of accuracy as follows; no discrimination (AUC $<0.5$ ), fairly acceptable $(0.5<$ AUC $<0.7)$, excellent $(0.7<$ AUC $<0.9)$ and outstanding $(0.9<\mathrm{AUC})$.

\section{Results}

In this section, we describe the result of each tasks: Marker selection and classification. For marker selection, we shows the performance of selected marker sets and analysis AUC values of combinations from single marker AUC values. For classification, we compare the three different classification methods.

\section{Marker selection results}

Table 1 shows the optimal combination lists for the size of combination. The first column in Table 1 indicates algorithm for marker selection. Each describes as follows: GA is genetic algorithm, RF is random forest and LR is logistic regression. The second column presents the number of combinations. The listed combinations which ranges from 2 to 4 , are selected to average AUC. Each combinations are chosen the highest AUC value from all possible combinations with the number of combination. The bold presents the highest AUC value in each algorithm.

The GA and RF yields 0.9 AUC value and 0.98 AUC value. With slight improvement, T-Test and LR perform 0.99 AUC value. In four results, GA have the lowest AUC value of 0.86 on 2 combination. The lowest performance among high score in each algorithm also perform in GA.

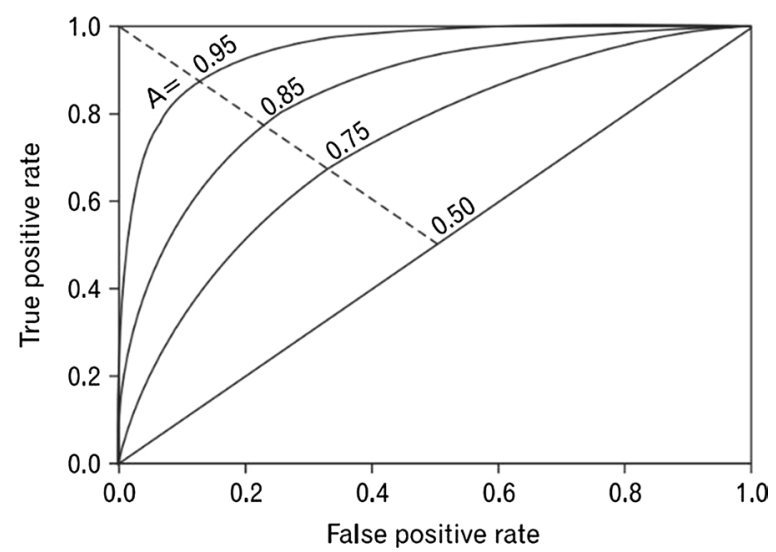

Fig. 1 Receiver operating characteristic area under the curve (ROC AUC). The number on each curve indicates the measure of diagnosis accuracy 
Table 1 The AUC value for each combinations for each feature selection algorithm

\begin{tabular}{llll}
\hline Algorithm & Size & Combinations & AUC \\
\hline GA & 2 & ApoCIII, TTR & 0.86 \\
& 3 & IL-6, CEA, OPN & 0.90 \\
& 4 & MIF, ApoAI, OPN, IL-6 & 0.90 \\
RF & 2 & CA125, HE4-ELISA & 0.92 \\
& 3 & Prolactin, TTR, HE4-ELISA & 0.98 \\
& 4 & TTR, Prolactin, CA125, HE4-ELISA & 0.98 \\
T-Test & 2 & TTR, ApoCIII & 0.95 \\
& 3 & TTR, ApoCIII, Prolactin & 0.98 \\
& 4 & TTR, ApoCIII, Prolactin, OPN & 0.99 \\
LR & 2 & Prolactin, TTR & 0.98 \\
& 3 & ApoCIII, HE4-ELISA, Prolactin & 0.99 \\
& 4 & HE4-ELISA, PDGF-AA, Prolactin, TTR & 0.99 \\
\hline
\end{tabular}

Table 2 Top 10 AUC value of single marker computed to logistic regression

\begin{tabular}{lc}
\hline Marker & AUC \\
\hline TTR & 0.94 \\
HE4-ELISA & 0.92 \\
Prolactin & 0.91 \\
CA125 & 0.88 \\
ApoCIII & 0.84 \\
MIF & 0.80 \\
OPN & 0.78 \\
PDGF-AA & 0.76 \\
IL-6 & 0.73 \\
CRP & 0.71 \\
\hline
\end{tabular}

Except RF, the rest of algorithm shows the best AUC value to 4 combinations. However, there are no major differentiation of AUC value between 3 and 4 marker combinations. Intuitively, we find that it is not necessary to use 4 marker combination.

Table 2 describes top 10 single markers sorted to AUC value. TTR, HE4-ELISA and Prolactin which have AUC value bigger than 0.9, are well captured to important biomarker when selecting the marker combination, except GA. We analysis that GA shows the low AUC compared to the rest of combinations, since combined markers have AUC value which is even less than 0.8 . Figure 2 shows ROC curve for the best 5 single marker.

\section{Classification}

Table 3 shows the accuracy of classifications for each marker combination. In marker sets selected by GA, 2 combination shows the best performance of 0.88 using KNN. The 3 combination performs the lowest accuracy of 0.81 in overall results. In marker sets chosen by RF, 3 and 4 combination yield almost same accuracy using LR. The high score of 2 combination and 3, 4 combination display a significant gap with 0.12. Marker sets chosen by T-Test also shows totally same performance of 0.95 using LR. In optimal marker combinations using LR, a classifier using LR yields 0.95 accuracy, which is same 


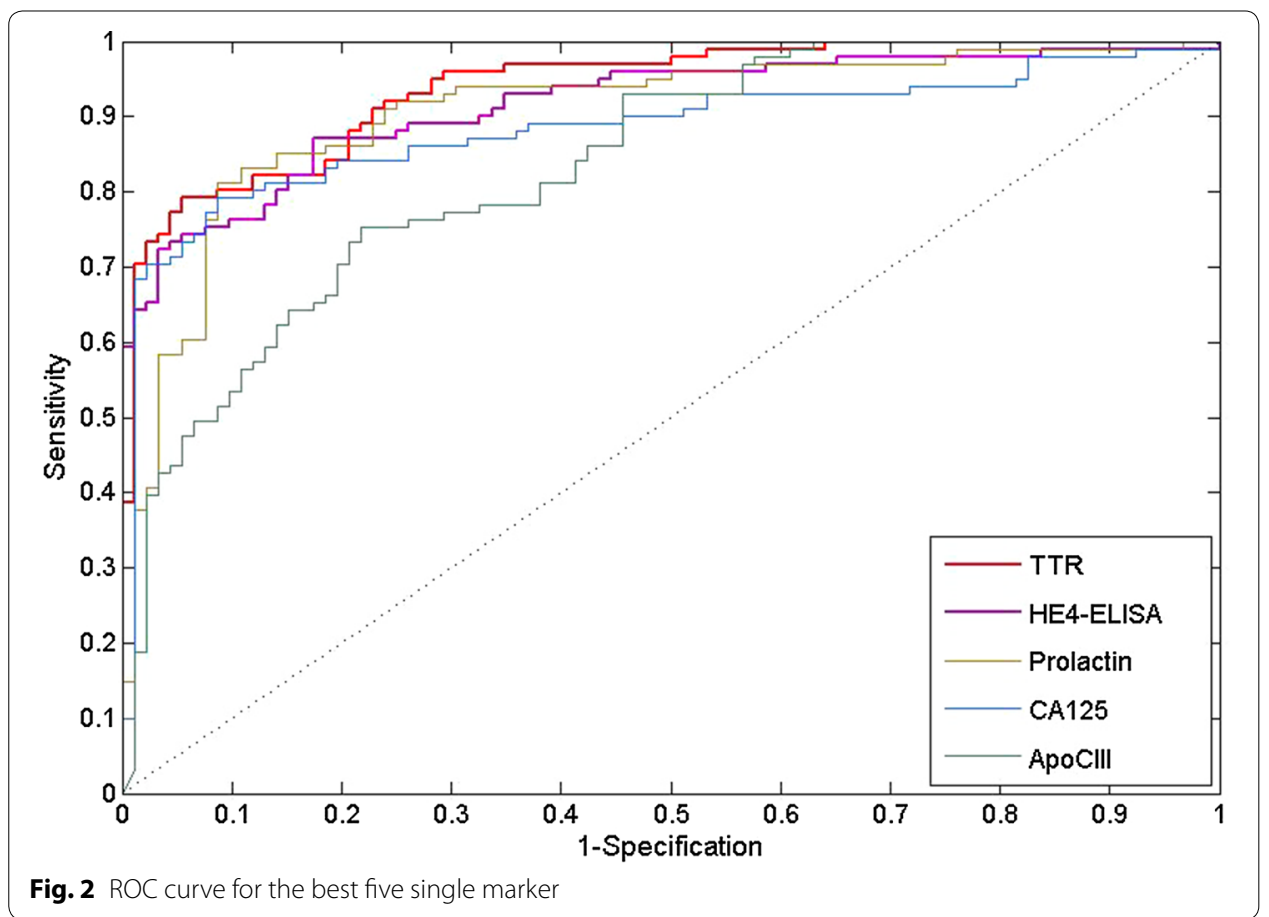

Table 3 Accuracy of three classification method for each marker combination

\begin{tabular}{lllll}
\hline Algorithm & Combinations & LDA & KNN & LR \\
\hline GA & ApoCIII, TTR & 0.88 & 0.88 & 0.87 \\
& IL-6, CEA, OPN & 0.67 & 0.79 & 0.81 \\
& MIF, APoAI, OPN, IL-6 & 0.74 & 0.86 & 0.83 \\
RF & CA125, HE4-ELISA & 0.69 & 0.83 & 0.78 \\
& Prolactin, TTR, HE4-ELISA & 0.91 & 0.93 & 0.95 \\
& TTR, Prolactin, CA125, HE4-ELISA & 0.92 & 0.91 & 0.94 \\
T-Test & TTR, ApoCIII & 0.88 & 0.88 & 0.87 \\
& TTR, ApoCIII, Prolactin & 0.93 & 0.93 & 0.95 \\
& TTR, ApoCIII, Prolactin, OPN & 0.93 & 0.93 & 0.95 \\
LR & Prolactin, TTR & 0.91 & 0.92 & 0.93 \\
& ApoCIII, HE4-ELISA, Prolactin & 0.93 & 0.94 & 0.93 \\
& HE4-ELISA, PDGF-AA, Prolactin, TTR & 0.92 & 0.94 & 0.95 \\
\hline
\end{tabular}

as T-Test. However, a classifier using logistic regression shows the stable performance across all combination size.

Not surprisingly, the GA algorithm which has a lowest AUC value for marker selection, performs the lowest accuracy of 0.80 for 3 combination. All marker selection algorithm except GA, shows better performance for the 3 and 4 combinations rather than 2 combinations. The performance between 2 combination and 4 combination for RF, T-Test and LR are about $0.11,0.6$ and 0.2 apart, respectively. The classifier using logistic regression shows the outstanding performance in over $70 \%$ of marker sets. We also find that TTR and Prolactin contains in combination which shows the good performance. 


\section{Discussion}

In this paper, we present the exploration for the marker selection and classification between cancer and normal samples, using machine learning algorithms. For marker selection, we find all methods except genetic algorithm, can capture in combining marker sets a marker, which has a high AUC value. Among them, logistic regression shows high performance for all combinations in general. For classification, logistic regression also presents the highest accuracy. Logistic regression also shows the stable accuracy on classification. It indicates that logistic regression can capture optimal combination and classify two difference class well. The experimental results shows that logistic regression is an outstanding algorithm for both problem.

\section{Conclusions}

We find the combination which contains TTR and Prolactin gives high performance for cancer detection. With the stability and accuracy, we can find Her-ELISA, PDGF-AA, Prolactin and TTR is the best biomarkers for classifying cancer samples from healthy to cancer data. Early detection of ovarian cancer can reduce high mortality rates. Finding a combination of multiple biomarkers for diagnostic tests with high sensitivity and specificity is very important. For future works, we can apply our approach to urine samples or can be considerer to highly influential factor for detecting ovarian cancer, such as age, the stage of cancer and the state of menopause.

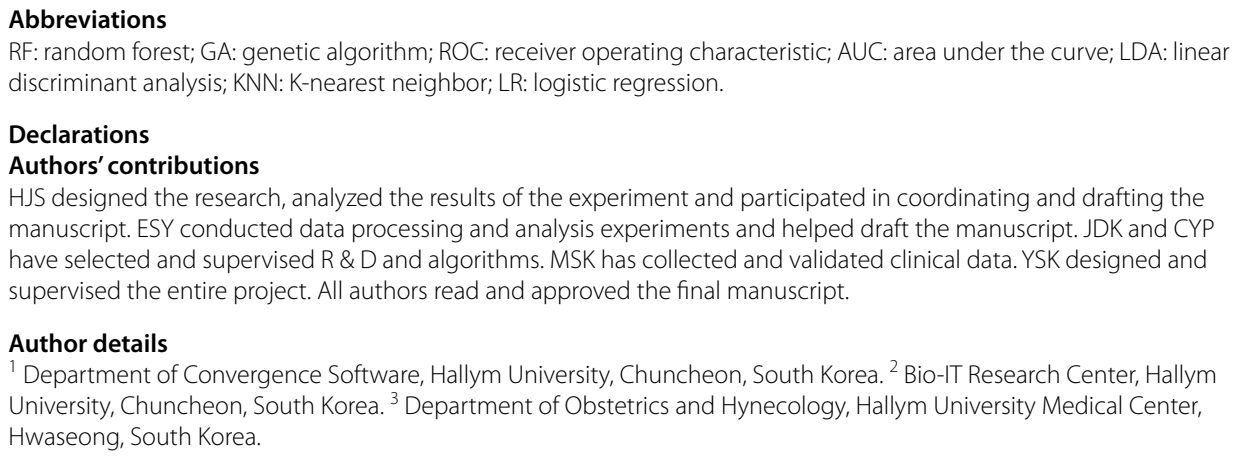
manuscript. ESY conducted data processing and analysis experiments and helped draft the manuscript. JDK and CYP have selected and supervised R \& D and algorithms. MSK has collected and validated clinical data. YSK designed and supervised the entire project. All authors read and approved the final manuscript.

\section{Author details}

${ }^{1}$ Department of Convergence Software, Hallym University, Chuncheon, South Korea. ${ }^{2}$ Bio-IT Research Center, Hallym University, Chuncheon, South Korea. ${ }^{3}$ Department of Obstetrics and Hynecology, Hallym University Medical Center, Hwaseong, South Korea.

Acknowledgements

The Ministry of Science, ICT and Future Planning (2013R1A1A3013037), Basic Science Research Program through the National Research Foundation of Korea(NRF).

Competing interests

The authors declare that they have no competing interests.

Availability of data and materials

The data analyzed during the study can be used if there is a reasonable request.

\section{Consent for publication}

None.

Ethics approval and consent to participate

This study was approved by the Institutional Review Board of Hallym University Hospital (No.2010-36).

Funding

Publication of this article was sponsored by Hallym University Research Fund 2015 (H20150664). 
available online at https://biomedical-engineering-online.biomedcentral.com/articles/supplements/volume-17-suppl ement-2.

\title{
Publisher's Note
}

Springer Nature remains neutral with regard to jurisdictional claims in published maps and institutional affiliations.

\author{
Published: 6 November 2018
}

\section{References}

1. Clarke CH, Yip C, Badgwell D, Fung ET, Coombes KR, Zhang Z, Bast RC. Proteomic biomarkers apolipoprotein A1, truncated transthyretin and connective tissue activating protein III enhance the sensitivity of CA125 for detecting early stage epithelial ovarian cancer. Gynecol Oncol. 2011;122(3):548-53.

2. Hensley ML, Martee L, Castiel M, Robson E. Screening for ovarian cancer: what we know, what we need to know. Oncology. 2000;14(11):1601-7.

3. Kim YS, Jang MK, Park CY, Song HJ, Kim JD. Exploring multiple biomarker combination by logistic regression for early screening of ovarian cancer. Int J Bio-Sci Bio-Technol. 2013;5(2):67-75.

4. Jang MK, Kim YS, Park CY, Song HJ, Kim HJ. Integration of menopausal information into the multiple biomarke diagnosis for early diagnosis of ovarian cancer. Int J Bio-Sci Bio-Technol. 2013:5(4):215-22.

5. Song HJ, Ko SK, Kim JD, Park CY, Kim YS. Looking for the optimal machine learning algorithm for the ovarian cancer screening. Int J Bio-Sci Bio-Technol. 2013;5(2):41-8.

6. Blackwell K, Lokshin AE. Serum biomarker profiles and response to neoadjuvant chemo therapy for locally advanced breast cancer. Breast Cancer Res. 2008;10:R45.

7. Nolen B, Marrangoni A, Velikokhatnaya L, Prosser D, Winans M, Gorelik E, Lokshin A. A serum based analysis of ovarian epithelial tumorigenesis. Gynecol Oncol. 2009;112:47-54.

8. Van Gorp T, Cadron I, Despierre E, Daemen A, Leunen K, Amant F, Vergote I. HE4 and CA125 as a diagnostic test in ovarian cancer: prospective validation of the risk of ovarian malignancy algorithm. Br J Cancer. 201 1;104(5):863-70.

9. Einhorn N, SjÖvall K, Knapp RC, Hall P, Scully RE, Robert C, Bast JR, Vincent R, Zurawski JV. Prospective evaluation of serum CA 125 levels for early detection of ovarian cancer. Obstet Gynecol. 1992;80(1):14-28.

10. Yin BW, Dnistrian A, Lloyd KO. Ovarian cancer antigen CA125 is encoded by the MUC16 mucin gene. Int J Cancer. 2002;98(5):737-40.

11. US Department of Health and Human Services. Draft Guidance for Industry, Clinical Laboratories, and Staff: In vitro diagnostic multivariate index assays, 2007. http://www.fda.gov/downloads/MedicalDevices/DeviceRegulationandG uidance/GuidanceDocuments/ucm071455.pdf. Accessed 20/02/2013.

12. Nolen B, Marrangoni A, Velikokhatnaya L, Prosser D, Winans M, Gorelik E, Lokshin A. A serum based analysis of ovarian epithelial tumorigenesis. Gynecol Oncol. 2009;112(1):47-54.

13. Kozak KR, Su F, Whitelegge JP, Faull K, Reddy S, Farias-Eisner R. Characterization of serum biomarkers for detection of early stage ovarian cancer. Proteomics. 2005;5(17):4589-96.

14. He G, Holcroft CA, Beauchamp MC, Yasmeen A, Ferenczy A, Kendall-Dupont J, Gotlieb WH. Combination of serum biomarkers to differentiate malignant from benign ovarian tumours. J Obstet Gynaecol Can. 2012;34(6):567-74.

15. Ho TK. Random decision forests. In: Proceedings of the third international conference. Vol. 1. Piscataway: IEEE; 1995. 278-82.

16. David GE, John HH. Genetic algorithms and machine learning. Mach Learn. 1988;3(2):95-9.

17. Cox DR. The regression analysis of binary sequences. J Roy Stat Soc Ser B (Methodol). 1958;20:215-42.

18. David BM, Andrew YN, Michael IJ. Latent Dirichlet allocation. J Mach Learn Res. 2003;3:993-1022.

19. Altman NS. An introduction to Kernel and nearest-neighbor nonparametric regression. Am Stat. 1992;46(3):175-85.

20. Swets JA. Measuring the accuracy of diagnostic systems. Science. 1988;240(4857):1285-93.

21. Greiner M, Pfeiffer D, Smith RD. Principles and practical application of the receiver operating characteristic analysis for diagnostic tests. Prev Vet Med. 2000;45(1):23-41.

Ready to submit your research? Choose BMC and benefit from:

- fast, convenient online submission

- thorough peer review by experienced researchers in your field

- rapid publication on acceptance

- support for research data, including large and complex data types

- gold Open Access which fosters wider collaboration and increased citations

- maximum visibility for your research: over $100 \mathrm{M}$ website views per year

At BMC, research is always in progress.

Learn more biomedcentral.com/submissions 